\title{
Robotic edge machining with abrasive brushes
}

\author{
Yuriy Dimov ${ }^{1}$, and Dmitriy Podashev ${ }^{2, *}$ \\ ${ }^{1}$ Irkutsk National Research Technical University, 83 Lermontov street, 664074, Irkutsk, Russia
}

\begin{abstract}
The article describes application of an industrial robot with an electric spindle for finish machining of parts made from aluminum alloy V95pchT2 and titanium alloy VT-20 using abrasive brushes. Effects of cutting velocity, brush deformation and supply on machining performance (edge size) and surface quality (positioning and roughness) have been identified. Based on the experimental data, mathematical dependencies have been developed.
\end{abstract}

Manual labor mechanization and automation in edge machining or surface cleaning are still crucial issues for aircraft, rocket and machine building. Edge machining operations can be either automated or manual. The latter method is time and labor consuming.

Edge machining with abrasive brushes was described in [1-5] which deal with the issues of effective utilization of abrasive brushes and their rational application areas and technological functions. Some researchers describe contacts of abrasive brushes with the surface. However, the issues of edge machining robotization have not been touched upon in published researches.

The present study seeks to analyze application of industrial robots for edge machining. The task is to study performance of edge machining with radial abrasive brushes and surface quality improvement methods.

For research purposes, we used KUKA KR 210 R2700 EXTRA with RC90 electric spindle by Elettromeccanica Giordano Colombo (Italy) and a bench (Forster, Germany) of 4000x1500 $\mathrm{mm}$ in size. Before installing, we used TD2009 Comfort Plus module balance system by Haimer (Germany) to balance the tool to G6,3 according to State Standard ISO 1940-1-2007.

Abrasive brushes are efficient edge machining tools. Currently, the brushes are produced by such companies as 3M (Minnesota Mining and Manufacturing Company), C. Hilzinger-Thum, Eisenblatter, Hoffmann Group, Lippert Unipol, August Ruggeberg Pferd-Werkzeuge, Osborn International, JAZ, Hommel Hercules Werkzeughandel, etc.

For research purposes, BB-ZB Type C (Fig. 1) and Type A (Fig. 2) radial bristle brushes produced by $3 \mathrm{M}$ were used. Scotch-Brite ${ }^{\mathrm{TM}}$ Bristle brushes are round polymeric slices with straight or curved flexible bristles. Bristles are plastic embedded with abrasive $\left(3 \mathrm{M}^{\mathrm{TM}} \mathrm{Cu}-\right.$ bitron $^{\mathrm{TM})}$ grit. The abrasive material is a precision pyramid-shaped crystal having a microcrystal structure. A brush of any width can be made of several discs (Fig. 1 and 2).

\footnotetext{
* Corresponding author: $\underline{\text { dbp } 90 @ \text { mail.ru }}$
} 


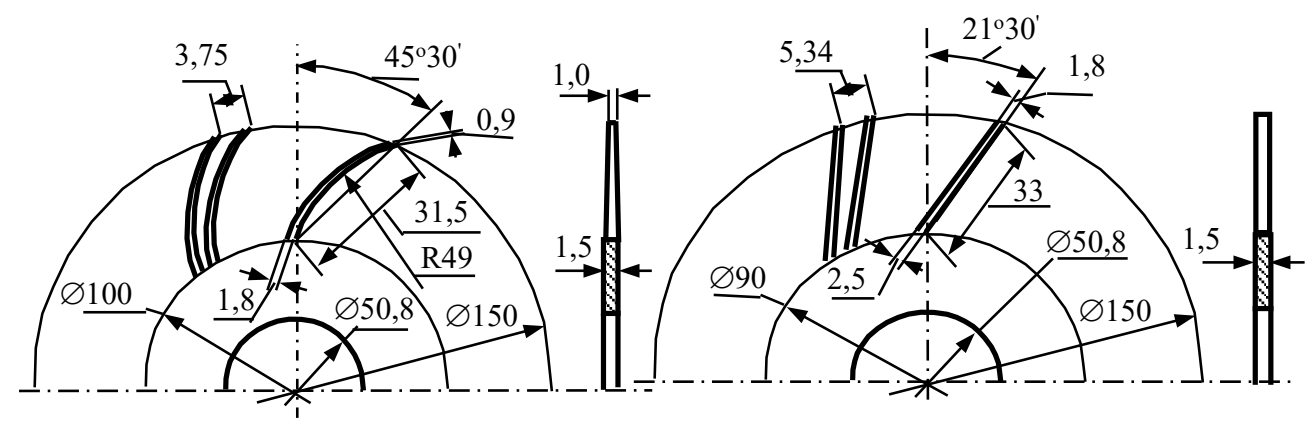

Fig. 1. BB-ZB Type C Brush Disc, 135 bristles Fig. 2. BB-ZB Type A Brush Disc, 90 bristles

Edge machining performance. Experiments were carried out on samples made from high tensile aluminum alloy V95pchT2 and Titanium alloy VT-20 using KUKA KR 210 R2700 EXTRA and BB-ZB Type A P50 and BB-ZB Type C P120 brushes.

The size of the machined edge $X, Y$ and p (Fig. 3) was measured using BMI-1C with a graduation mark of $1 \mu \mathrm{m}$.

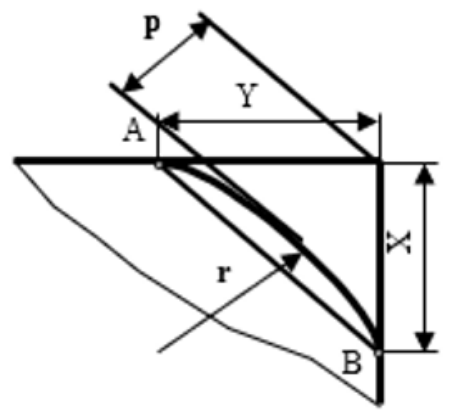

Fig. 3. Schemes used to determine rounding parameters.

Edge machining performance was assessed by a mean value of the $\mathrm{X}$-axis and $\mathrm{Y}$-axis edge size which is calculated by formula

$$
Z_{k}=\frac{X+Y}{2}
$$

where $X, Y$ are the coordinates of the transition of the rounding end into the straight line (see Fig. 3).

The effects of cutting rate $\mathrm{V}$, brush deformation $\Delta \mathrm{Y}$ (Fig. 4) and supply $\mathrm{S}$ are shown in Figures 5, 6 and 7 .
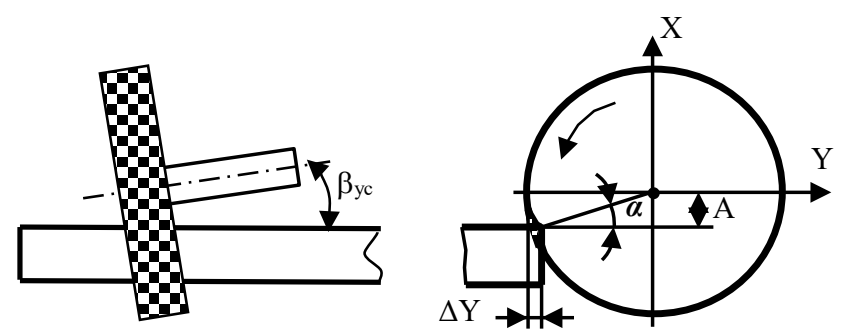

Fig. 4. Schemes for determining parameters $\alpha, \Delta Y$ and $\beta$ 

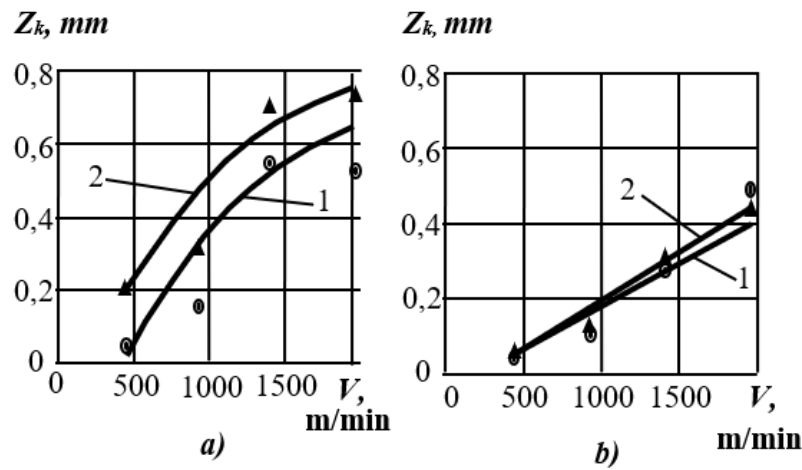

Fig.5. Dependencies of $Z_{k}$ on the cutting speed of the radial brush at $\Delta Y=3 \mathrm{~mm} ; \beta=10^{\circ}$ : a - for V95pchT2 at $\mathrm{S}=400 \mathrm{~mm} / \mathrm{min} ; \mathrm{b}-$ for VT- 20 at $\mathrm{S}=200 \mathrm{~mm} / \mathrm{min}: 1-\mathrm{BB}-\mathrm{ZB}$ Type A P50 at $\mathrm{A}=0 \mathrm{~mm}$; 2 - BB-ZB Type C P120 at A=-10 mm.
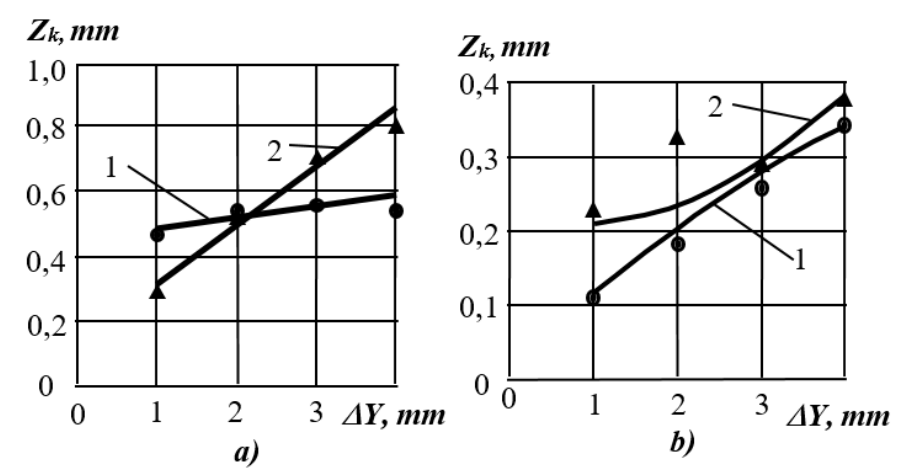

Fig.6. Dependencies of $Z_{k}$ on the radial brush deformation at $V=1413,72 \mathrm{~m} / \mathrm{min} ; \beta=10^{\circ}$ : a - for V95pchT2 at $\mathrm{S}=400 \mathrm{~mm} / \mathrm{min} ; \mathrm{b}-$ for VT- 20 at $\mathrm{S}=200 \mathrm{~mm} / \mathrm{min}: 1-\mathrm{BB}-\mathrm{ZB}$ Type A P50 at $\mathrm{A}=0 \mathrm{~mm}$; 2 - BB-ZB Type C P120 at A=-10 mm.

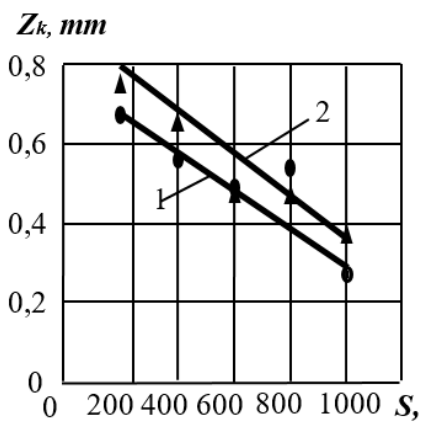

a)

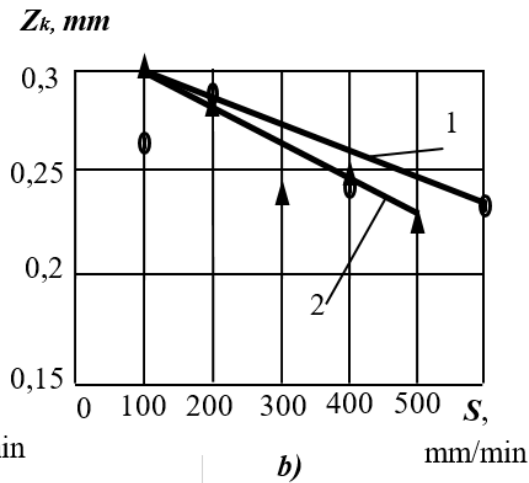

b)

Fig.7. Dependencies of $Z_{k}$ on the radial brush supply at $V=1413,72 \mathrm{~m} / \mathrm{min} ; \Delta Y=3 \mathrm{~mm} ; \beta=10^{\circ}$ : a - for V95pchT2; b - for VT-20: 1 - BB-ZB Type A P50 at A=0 mm; 2 - BB-ZB Type C P120 at A=-10 $\mathrm{mm}$. 
With increasing brush deformation (see Fig. 6), the edge size increases for V95pchT2 and VT-20 due to the increasing acting force [6].

The effect of supply on machining performance is shown in Figure 7. With increasing supply, the edge size decreases. It is easy to control changes in supply to get required edge sizes.

Using the experimental data, an equation for calculating edge size $Z_{k}$ in relation to velocity $\mathrm{V}$, brush deformation $\Delta \mathrm{Y}$, supply $\mathrm{S}$ and brush axle angle $\beta$ can be calculated

When machining long parts such as profiles in aircraft building, $\beta$ is required to eliminate contacts of the spindle head and a machined part.

$$
\begin{gathered}
Z_{k}=\left(a_{1} \cdot \Delta Y^{2}+a_{2} \cdot V^{2}+a_{3} \cdot S^{2}+a_{4} \cdot \Delta Y+a_{5} \cdot V+a_{6} \cdot S+a_{7} \cdot \Delta Y \cdot V+a_{8} \cdot \Delta Y \cdot S+a_{9} \cdot V \cdot S+a_{10} \cdot \Delta Y \cdot V \cdot S+a_{11}\right) \\
\cdot\left(a_{12} \cdot \beta^{3}+a_{13} \cdot \beta^{2}+a_{14} \cdot \beta+a_{15}\right)
\end{gathered}
$$

Table 1 shows the values of ratios $\boldsymbol{a}_{1-10}$, and $\boldsymbol{a}_{12}-\boldsymbol{a}_{14}$ and constant terms $\boldsymbol{a}_{11}$ and $\boldsymbol{a}_{15}$ in the equation.

\begin{tabular}{|c|c|c|c|c|c|}
\hline \multirow{2}{*}{\multicolumn{2}{|c|}{$\begin{array}{c}\text { Material } \\
\text { Tool }\end{array}$}} & \multicolumn{2}{|c|}{ V95pchT2 } & \multicolumn{2}{|c|}{ VT20 } \\
\hline & & \multirow{2}{*}{$\begin{array}{c}\text { BB-ZB Type A } \\
\text { P50 } \\
-0,01 \\
\end{array}$} & \multirow{2}{*}{$\begin{array}{c}\text { BB-ZB Type C } \\
\text { P120 } \\
-0,0175 \\
\end{array}$} & \multirow{2}{*}{$\begin{array}{c}\begin{array}{c}\text { BB-ZB Type A } \\
\text { P50 }\end{array} \\
-5 \cdot 10^{-3}\end{array}$} & \multirow{2}{*}{$\begin{array}{c}\begin{array}{c}\text { BB-ZB Type C } \\
\text { P120 }\end{array} \\
0,0175\end{array}$} \\
\hline \multirow{15}{*}{ 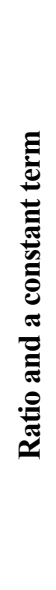 } & $a_{1}$ & & & & \\
\hline & $a_{2}$ & $-2 \cdot 10^{-7}$ & $-1,6 \cdot 10^{-7}$ & $1,5 \cdot 10^{-8}$ & $2 \cdot 10^{-8}$ \\
\hline & $a_{3}$ & $-6,5 \cdot 10^{-8}$ & $-2 \cdot 10^{-8}$ & $2 \cdot 10^{-7}$ & $1,5 \cdot 10^{-7}$ \\
\hline & $a_{4}$ & 0,082 & 0,2565 & 0,103 & $-0,0325$ \\
\hline & $a_{5}$ & $9 \cdot 10^{-4}$ & $7,8 \cdot 10^{-4}$ & $2,1 \cdot 10^{-4}$ & $2,1 \cdot 10^{-4}$ \\
\hline & $a_{6}$ & $-3,5 \cdot 10^{-4}$ & $-5 \cdot 10^{-4}$ & $-3 \cdot 10^{-4}$ & $-3 \cdot 10^{-4}$ \\
\hline & $a_{7}$ & $1,5 \cdot 10^{-9}$ & $1,5 \cdot 10^{-9}$ & $1,5 \cdot 10^{-9}$ & $1,5 \cdot 10^{-9}$ \\
\hline & $a_{8}$ & $-2,41 \cdot 10^{-9}$ & $-2,41 \cdot 10^{-9}$ & $-2,41 \cdot 10^{-9}$ & $-2,41 \cdot 10^{-9}$ \\
\hline & $a_{9}$ & $5,8 \cdot 10^{-11}$ & $5,8 \cdot 10^{-11}$ & $5,8 \cdot 10^{-11}$ & $5,8 \cdot 10^{-11}$ \\
\hline & $a_{10}$ & $1,5 \cdot 10^{-11}$ & $1,5 \cdot 10^{-11}$ & $1,5 \cdot 10^{-11}$ & $1,5 \cdot 10^{-11}$ \\
\hline & $a_{11}$ & $-0,3$ & $-0,52$ & $-0,26$ & $-0,06$ \\
\hline & $a_{12}$ & $9,143 \cdot 10^{-8}$ & $1,529 \cdot 10^{-6}$ & $1,829 \cdot 10^{-8}$ & $1,051 \cdot 10^{-6}$ \\
\hline & $a_{13}$ & $-6,24 \cdot 10^{-5}$ & $-1,368 \cdot 10^{-4}$ & $-5,95 \cdot 10^{-5}$ & $-1,295 \cdot 10^{-4}$ \\
\hline & $a_{14}$ & $-0,013985$ & $-0,016085$ & $-0,012379$ & $-0,01481$ \\
\hline & $a_{15}$ & 1,146 & 1,173 & 1,13 & 1,16 \\
\hline
\end{tabular}

Table 1. The values of ratios and a constant term in (2)

At given $Z_{k}, \Delta Y, V$ and $\beta$, supply $S$ is calculated by formula (3)

$$
S=\frac{-d+\sqrt{d^{2}-4 a_{3} \cdot f}}{2 a_{3}}
$$

where $d=a_{6}+a_{8} \cdot \Delta Y+a_{9} \cdot V+a_{10} \cdot \Delta Y \cdot V$;

$f=a 1 \cdot(\Delta Y)^{2}+a 2 \cdot V^{2}+a 4 \cdot \Delta Y+a 5 \cdot V+a 7 \cdot \Delta Y \cdot V+a 11-\left(\frac{z k}{a_{12} \cdot \beta^{3}+a_{13} \cdot \beta^{2}+a_{14} \cdot \beta+a_{15}}\right)$.

Surface quality. Surface quality depends on a positioning tolerance and a roughness (Ra). Positioning tolerance is a relative deviation from symmetric location of the rounding radius (Fig. 3):

$$
\delta=\frac{X-Y}{Z_{k}} .
$$

There are a rounding radius tolerance and $\delta$ tolerance.

For example, in aircraft building, according to Industrial Standard 1.000.22 - 80, edge rounding is $0,5 \mathrm{~mm}$ with a tolerance of $\pm 0,2 \mathrm{~mm}$. According to State Standard 30893.1 2002 
(ISO 2768-1 - 89), tolerance extremes for truncated edges (external rounding radii and bevel height) are as follows:

- for first-order (f) and mean ( $\mathbf{m})$ accuracy classes - $\pm 0,2 \mathrm{~mm}$;

- for coarse (c) and rough (v) accuracy classes - $\pm 0,4 \mathrm{~mm}$.

For accuracy classes f and $\mathbf{m}, \boldsymbol{\delta}$ is calculated by formula

$$
\delta=\frac{T}{\bar{X}_{\text {min }}}=\frac{0,4}{0,3}=1,33
$$

where $\mathrm{T}$ is the rounding radius tolerance; $\bar{X}_{\min }$ is the minimum limit of a radius size.

For accuracy classes $\mathbf{c}$ and $\mathrm{v}, \delta=0,8 / 0,3=2,66$.

In previous studies on positioning tolerances $\delta[7,8]$, patterns of the impact of brush deformation $\Delta \mathrm{Y}$, brush offsetting angle $\alpha$ (Fig. 4) and brush axle angle $\beta$ on the surface quality were analyzed.

As a result of the statistical analysis of experimental data, the relation between $\delta, \Delta \mathrm{Y}$ and $\alpha$ and $\beta$ was determined.

$\delta=a_{1} \cdot \alpha^{2}+a_{2} \cdot \beta^{2}+a_{3} \cdot \Delta Y^{2}+a_{4} \cdot \alpha+a_{5} \cdot \beta+a_{6} \cdot \Delta Y+a_{7} \cdot \beta \cdot \alpha+a_{8} \cdot \Delta Y \cdot \alpha+a_{9} \cdot \Delta Y \cdot \beta+a_{10} \cdot \beta \cdot \alpha \cdot \Delta Y+a_{11} \cdot(5)$

Values of ratios and constant terms are presented in Table 2.

Table 2. Values of ratios and constant terms in (5)

\begin{tabular}{|c|c|c|c|c|c|c|c|c|c|}
\hline \multirow{3}{*}{\multicolumn{2}{|c|}{$\begin{array}{c}\text { Material } \\
\text { Brush } \\
\text { Formula }\end{array}$}} & \multicolumn{4}{|c|}{ V95pchT2 } & \multicolumn{4}{|c|}{ VT20 } \\
\hline & & \multicolumn{2}{|c|}{ BB-ZB Type A P50 } & \multicolumn{2}{|c|}{$\begin{array}{c}\text { BB-ZB Type C } \\
\text { P120 }\end{array}$} & \multicolumn{2}{|c|}{ BB-ZB Type A P50 } & \multicolumn{2}{|c|}{$\begin{array}{c}\text { BB-ZB Type C } \\
\text { P120 }\end{array}$} \\
\hline & & $\delta$ & $\mathbf{R a}$ & $\delta$ & $\mathbf{R a}$ & $\delta$ & $\mathbf{R a}$ & $\delta$ & $\mathbf{R a}$ \\
\hline \multirow{11}{*}{ 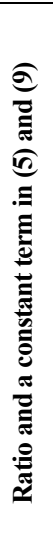 } & $a_{1}$ & $1,5 \cdot 10^{-4}$ & $-0,15$ & $1,52 \cdot 10^{-4}$ & $-0,075$ & $1,4 \cdot 10^{-4}$ & $9 \cdot 10^{-16}$ & $2,2 \cdot 10^{-4}$ & $-0,0125$ \\
\hline & $a_{2}$ & $8,3 \cdot 10^{-5}$ & $-3 \cdot 10^{-7}$ & $1,06 \cdot 10^{-4}$ & $8 \cdot 10^{-7}$ & $1,46 \cdot 10^{-5}$ & $-4,5 \cdot 10^{-7}$ & $-5 \cdot 10^{-6}$ & $1 \cdot 10^{-8}$ \\
\hline & $a_{3}$ & $-0,017$ & $-4,1 \cdot 10^{-7}$ & $5,35 \cdot 10^{-3}$ & $-5,5 \cdot 10^{-7}$ & $8 \cdot 10^{-3}$ & $6 \cdot 10^{-8}$ & $-6,68 \cdot 10^{-7}$ & $9 \cdot 10^{-7}$ \\
\hline & $a_{4}$ & $7,8 \cdot 10^{-4}$ & 1,47 & $7,81 \cdot 10^{4}$ & 0,585 & $1,92 \cdot 10^{-4}$ & 0,1 & $1 \cdot 10^{-3}$ & 0,178 \\
\hline & $a_{5}$ & $2,46 \cdot 10^{-3}$ & $1,3 \cdot 10^{-3}$ & $1,5 \cdot 10^{-4}$ & $-8 \cdot 10^{4}$ & $2,46 \cdot 10^{-3}$ & $1,62 \cdot 10^{-3}$ & $2,5 \cdot 10^{-4}$ & $2 \cdot 10^{-4}$ \\
\hline & $a_{6}$ & $-0,035$ & $-2,65 \cdot 10^{-3}$ & 0,02253 & $-6,5 \cdot 10^{-4}$ & $-3,46 \cdot 10^{-3}$ & $-1,4 \cdot 10^{-3}$ & $-4,32 \cdot 10^{-4}$ & $-1,6 \cdot 10^{-3}$ \\
\hline & $a_{7}$ & $2 \cdot 10^{4}$ & $1,5 \cdot 10^{-9}$ & $2 \cdot 10^{-4}$ & $1,5 \cdot 10^{-9}$ & $2 \cdot 10^{-4}$ & $1,5 \cdot 10^{-9}$ & $2 \cdot 10^{-4}$ & $1,5 \cdot 10^{-9}$ \\
\hline & as & $9,16 \cdot 10^{-4}$ & $-2,41 \cdot 10^{-9}$ & $1,52 \cdot 10^{-3}$ & $-2,41 \cdot 10^{-9}$ & $-1,97 \cdot 10^{-4}$ & $-2,41 \cdot 10^{-9}$ & $-1,04 \cdot 10^{4}$ & $-2,41 \cdot 10^{-9}$ \\
\hline & $a_{9}$ & $3,33 \cdot 10^{-5}$ & $5,8 \cdot 10^{-11}$ & $3,33 \cdot 10^{-5}$ & $5,8 \cdot 10^{-11}$ & $3,33 \cdot 10^{-5}$ & $5,8 \cdot 10^{-11}$ & $3,33 \cdot 10^{-5}$ & $5,8 \cdot 10^{-11}$ \\
\hline & $a_{10}$ & $2,7 \cdot 10^{-6}$ & $1,5 \cdot 10^{-11}$ & $2,7 \cdot 10^{-6}$ & $1,5 \cdot 10^{-11}$ & $2,7 \cdot 10^{-6}$ & $1,5 \cdot 10^{-11}$ & $2,7 \cdot 10^{-6}$ & $1,5 \cdot 10^{-11}$ \\
\hline & $a_{11}$ & $-1,05$ & $-0,29$ & $-2,44$ & 0,95 & $-1,02$ & 0,31 & -2 & 0,36 \\
\hline
\end{tabular}

$\boldsymbol{\delta}=0$ is optimum. To obtain this value, it is necessary to calculate angle $\boldsymbol{\alpha}$ using equation (5) (at given $\Delta \mathrm{Y}, \beta$ and $\delta=0$ ) by formula:

$$
\alpha=\frac{-b+\sqrt{b^{2}-4 a_{1} \cdot c}}{2 a_{1}}
$$

where

$b=a_{4}+a_{7} \cdot \beta+a_{8} \cdot \Delta Y+a_{10} \cdot \beta \cdot \Delta Y ; c=a_{2} \cdot \beta^{2}+a_{3} \cdot(\Delta Y)^{2}+a_{5} \cdot \beta+a_{6} \cdot \Delta Y+a_{9} \cdot \beta \cdot \Delta Y+a_{11}-\delta$.

For edge machining using KUKA KR 210 R2700 EXTRA with BB-ZB Type A P50 radial brush at $\alpha=0$, the real value of $\delta$ varies between $+0,442$ and $-0,212$; for edge machining with 
BB-ZB Type C P120 radial brush at $\alpha=10^{\circ}, \delta$ varies between $+0,919$ and $+0,27$. These tolerances are admissible.

To obtain equal sizes of edges $\boldsymbol{Z}_{\boldsymbol{k}}$ on horizontal and angled segments with $\boldsymbol{\beta}$, the sequence of machining modes should be as follows:

1. Velocity $\boldsymbol{V}$ and brush deformation $\Delta \mathbf{Y}$ meet technological recommendations.

2. Angle $\boldsymbol{\alpha}$ is calculated by formula (6) at $\boldsymbol{\beta}=0$.

3. Supply $\mathbf{S}$ is calculated by formula (2) at $\boldsymbol{\beta}=0$, by formula (3) - for a horizontal segment in order to achieve size $\boldsymbol{Z}_{\boldsymbol{k}}$.

4. Angle $\boldsymbol{\alpha}$ is calculated by formula (6) at angle $\boldsymbol{\beta}$ and brush deformation $\Delta \mathbf{Y}$ for the angled surface.

5. Supply $\boldsymbol{S}$ along the angled surface is calculated by formula (2) at given $\boldsymbol{Z}_{\boldsymbol{k}}$ and $\boldsymbol{\beta}$.

6. Horizontal $\boldsymbol{S}_{\Gamma}$ and vertical $\boldsymbol{S}_{\text {B }}$ supply components are calculated by formulas

$$
S_{\Gamma}=S \cdot \cos \beta, \quad S_{\mathrm{B}}=S \cdot \sin \beta .
$$

As can be seen, supply $\boldsymbol{S}$ is larger for machining of horizontal surfaces $(\boldsymbol{\beta}=0)$ than angled ones $\left(\boldsymbol{S}_{\boldsymbol{\Gamma}}\right.$ ). To achieve a target edge size (e.g., $\left.\boldsymbol{Z}_{\boldsymbol{k}}=0,5 \mathrm{~mm}\right)$, it is necessary to substitute $\mathbf{S}$ for $\boldsymbol{S}_{\boldsymbol{\Gamma}}$ or increase brush deformation $\Delta \mathbf{Y}$ according to equation (2).

At given $\boldsymbol{Z}_{\boldsymbol{k}}, \boldsymbol{V}, \boldsymbol{S}$ and $\boldsymbol{\beta}$, deformation $\boldsymbol{\Delta} \boldsymbol{Y}$ is calculated by formula:

$$
\Delta Y=\frac{-g+\sqrt{g^{2}-4 a_{1} \cdot t}}{2 a_{1}}
$$

where $g=a_{4}+a_{7} \cdot V+a_{8} \mathrm{~S}+a_{10} \cdot V \cdot S$;

$$
t=a_{2} \cdot V^{2}+a_{3} \cdot S^{2}+a_{5} \cdot V+a_{6} \cdot S+a_{7} \cdot \Delta Y \cdot V+a_{11}-\left(\frac{z k}{a_{12} \cdot \beta^{3}+a_{13} \cdot \beta^{2}+a_{14} \cdot \beta+a_{15}}\right) .
$$

Edge roughness was measured with Bruker Contour GT-K1.

For edge machining with BB-ZB Type A P50 и BB-ZB Type C P120 radial brushes, surface roughness Ra depends on cutting velocity $\mathrm{V}$, brush deformation $\triangle \mathrm{Y}$ and supply $\mathrm{S}$ (Fig. 8, 9 and 10).
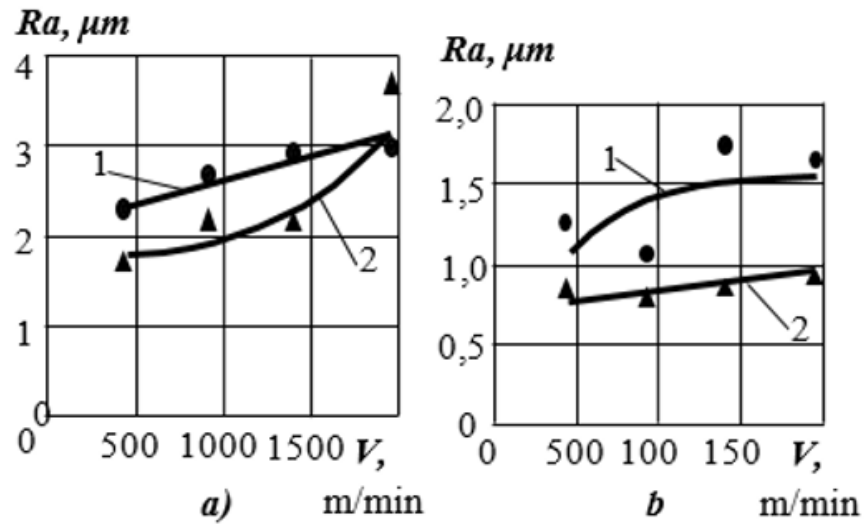

Fig.8. Dependencies of Ra on the cutting speed at $\Delta Y=3 \mathrm{~mm} ; \beta=10^{\circ}$ : a - for V95pchT2 at $S=400$ $\mathrm{mm} / \mathrm{min} ; \mathrm{b}$ - for VT-20 at S=200 mm/min: 1 - BB-ZB Type A P50 at A=0 mm; 2 - BB-ZB Type C $\mathrm{P} 120$ at $\mathrm{A}=-10 \mathrm{~mm}$. 

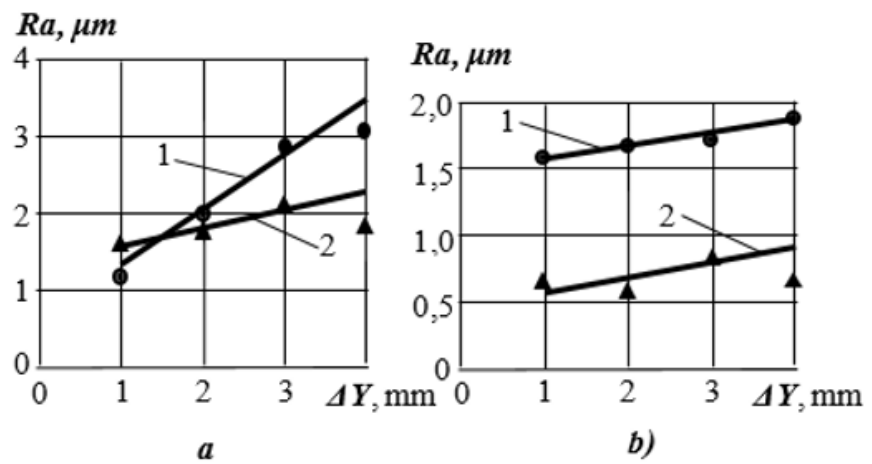

Fig.9. Dependencies of Ra on the radial brush deformation at $V=1413,72 \mathrm{~m} / \mathrm{min} ; \beta=10^{\circ}$ : $\mathrm{a}$ - for V95pchT2 at $\mathrm{S}=400 \mathrm{~mm} / \mathrm{min} ; \mathrm{b}-$ for VT-20 at $\mathrm{S}=200 \mathrm{~mm} / \mathrm{min}: 1-\mathrm{BB}-\mathrm{ZB}$ Type A P50 at A=0 mm; 2 - BB-ZB Type C P120 at A=-10 mm.

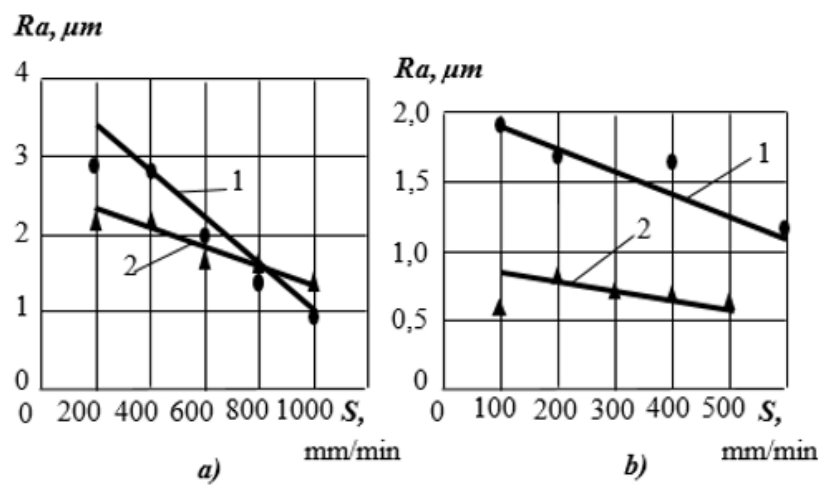

Fig.10. Dependencies of $R a$ on the radial brush supply at $V=1413,72 \mathrm{~m} / \mathrm{min} ; \Delta Y=3 \mathrm{~mm} ; \beta=10^{\circ}$ : a for V95pchT2; $\mathrm{b}$ - for VT-20: 1 - BB-ZB Type A P50 at A=0 mm; 2 - BB-ZB Type C P120 at A=$10 \mathrm{~mm}$.

With increasing velocity and deformation values, roughness increases [6] due to increasing interaction of the edge and brush bristles.

Based on the statistical analysis of experimental data, we obtained an equation for calculating a roughness parameter depending on velocity $\mathrm{V}$, brush deformation $\Delta \mathrm{Y}$ and supply $\mathrm{S}$.

$$
R a=a_{1} \cdot \Delta Y^{2}+a_{2} \cdot V^{2}+a_{3} \cdot S^{2}+a_{4} \cdot \Delta Y+a_{5} \cdot V+a_{6} \cdot S+a_{7} \cdot \Delta Y \cdot V+a_{8} \cdot \Delta Y \cdot S+a_{9} \cdot V \cdot S+a_{10} \cdot \Delta Y \cdot V \cdot S+a_{11} .
$$

Values of ratios $\boldsymbol{a}_{1-10}$, and a constant term $\boldsymbol{a}_{11}$ in the equation are presented in Table 2.

The experiments are relevant for edges which are available for machining on horizontal and angled (up to $60^{\circ}$ ) surfaces.

Examples of edge machining on different surfaces. Radius edge machining with radial abrasive brushes can be performed in two ways.

1. Machining by rotating a spindle head with a required radius based on the developed program.

2. Machining without a spindle head. It is a two-stage operation (see Fig. 11). At the first stage, segment A-B is machined at simultaneous horizontal and vertical supplies. Vertical supply $S_{B}$ determined by equations (3) and (7) and brush deformation $\Delta Y$ determined by (8) are calculated with regard to changing angle $\beta$ 


$$
\begin{gathered}
\beta=\beta_{y c}+\gamma, \\
S_{B R}=S_{B}+\left(\Delta Y_{R}-\Delta Y_{y c}\right)
\end{gathered}
$$

where $\Delta \mathrm{Y}_{\mathrm{R}}$ is the brush deformation determined by (8) at $\beta$ determined by (10) at current value $\gamma ; \Delta \mathrm{Y}_{\mathrm{fix}}$ is the brush deformation determined by (8) at fixed value $\beta_{\mathrm{fix}}$. At the second stage, segment B-C is machined after cornering the head rotation. Vertical supply $\mathrm{S}_{\mathrm{B}}$ (3) (7) and brush deformation $\Delta \mathrm{Y}(8)$ are calculated with regard to changing angle $\beta$ by (10).

$$
\mathrm{S}_{\mathrm{BR}}=\mathrm{S}_{\mathrm{B}}-\left(\Delta \mathrm{Y}_{\mathrm{R}}-\Delta \mathrm{Y}_{\mathrm{yc}}\right) \text {, }
$$

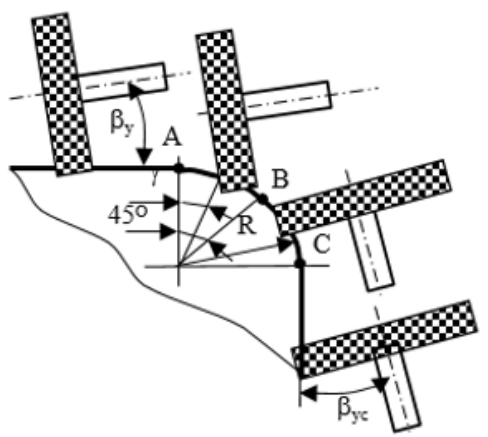

Fig.11. Scheme of radial edge machining.

When edge machining in oval holes, it is necessary to avoid contacts with the opposite side of the edge. To this end, it is necessary to determine permissible hole width B (Fig. 12) at specified brush deformation $\Delta \mathrm{Y}$, i.e. the size of $\mathrm{B}$ has to be $\mathrm{B} \geq \mathrm{M}$.

$$
\begin{gathered}
M=\frac{L}{2}+A \\
\text { where }=2 \cdot \sqrt{2 \cdot \Delta Y \cdot R-\Delta Y^{2}} ; A=R \cdot \sin \alpha .
\end{gathered}
$$

Here $\Delta Y=R-\sqrt{R^{2}-0,25 L^{2}}$.

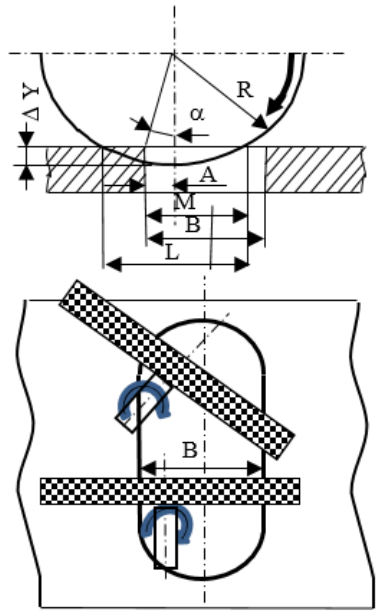

Fig.12. Edge machining scheme for an oval hole

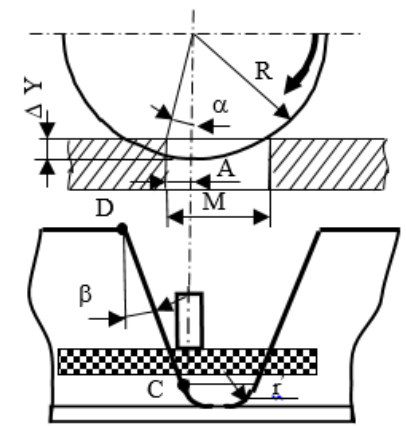

Fig.13. Scheme of angled surface machining with a radial brush 
Solving equations (13) and (14), we have

$$
M=A+\sqrt{2 \Delta Y \cdot R-\Delta Y^{2}} .
$$

As can be seen from (16), the permissible width of an oval hole depends on brush deformation $\Delta Y$ and its radius $R$. If it is impossible to change the brush radius, the preload of $\Delta Y$ should be changed by formula

$$
\Delta Y=R-\sqrt{R^{2}-(M-A)^{2}} .
$$

Radius edge machining can be performed by rotating the spindle head or in two stages (see Fig. 11).

Table 3 presents machining variants for narrow slots with width B using BB-ZB Type A P50 and BB-ZB Type C P120 radial brushes. Depending on a slot width, the target can be achieved by changing the brush preload or type.

Edge machining on straight segments with angled surfaces (with $\beta \leq 60^{\circ}$ ) contacting with a vertical wall (Fig. 13). The brush should be parallel to the vertical wall. Based on the suggested method, with a chosen brush type and known angle $\beta$, it is not difficult to calculate the value of deformation $\Delta \mathrm{Y}$.

Table 3. Minimum permissible slot widths when machining edges of V95pchT2 samples with radial

\begin{tabular}{|c|c|c|c|c|c|c|c|c|}
\hline $\begin{array}{c}\text { Brush axle } \\
\text { angle }\end{array}$ & \multicolumn{4}{|c|}{$\beta=0$} & \multicolumn{4}{|c|}{$\beta=10^{\circ}$} \\
\hline Brush & $\begin{array}{l}\Delta \mathbf{Y}, \\
\mathbf{m m}\end{array}$ & $\alpha,{ }^{0}$ & $\mathbf{A}, \mathbf{m m}$ & $\mathbf{M}, \mathbf{m m}$ & $\begin{array}{c}\Delta \mathbf{Y}_{\mathrm{cp}}, \\
\mathbf{m m}\end{array}$ & $\alpha,^{0}$ & $\mathbf{A}, \mathbf{m m}$ & $\mathbf{M}, \mathbf{m m}$ \\
\hline \multirow{3}{*}{$\begin{array}{c}\text { BB-ZB } \\
\text { Type A } \\
\text { P50 }\end{array}$} & 2 & $-9,287$ & $-12,104$ & 5,101 & 2 & \multirow{3}{*}{0} & \multirow{3}{*}{0} & 17,205 \\
\hline & 3 & $-7,641$ & $-9,973$ & 11,027 & 4 & & & 24,166 \\
\hline & 4 & $-4,983$ & $-6,514$ & 17,652 & 6 & & & 29,394 \\
\hline \multirow{3}{*}{$\begin{array}{l}\text { BB-ZB } \\
\text { Type C } \\
\text { P120 }\end{array}$} & 2 & $+22,912$ & $\begin{array}{l}+29,177 \\
\end{array}$ & 46,404 & 2 & \multirow{3}{*}{13,3} & \multirow{3}{*}{17,254} & 34,458 \\
\hline & 3 & $+17,242$ & $+22,231$ & 43,231 & 4 & & & 41,420 \\
\hline & 4 & $+11,485$ & $+14,933$ & 39,100 & 6 & & & 46,648 \\
\hline
\end{tabular}
bushes of $\varnothing 150 \mathrm{~mm}$ in size

\section{Conclusion}

1. Edge machining using robots with electric spindles can be used instead of manual edge rounding.

2. The experiments showed that edge machining performance and surface quality can have different values depending on the cutting velocity, brush deformation and supply. Target surface quality and high edge machining performance values can be obtained by controlling these parameters.

3. Technological functions of the robot can be expanded by varying elastic abrasive tools (radial and pitch brushes, flap wheels, nonwoven abrasive wheels, etc.). and sizes.

\section{References}

1. Yu.D. Abrashkevich, G.M. Machishin. Effective utilization of an abrasive brush, Bulletin of Kharkov National Automobile Road University, v. 73, pp. 59-62 (2016)

2. G.M. Machishin. Identification of a rational area of application of a polymer abrasive tool, Bulletin of Kharkov National Automobile Road University, v. 65-66, pp. 117-122 (2014)

3. B.E. Pini, D.R. Yakovlev. On technological abilities of polymer abrasive brushes, Proceedings of Moscow State Technical University MAMI, v. 1 (7), pp. 148-151 (2009) 
4. D.R. Yakovlev, B.E. Pini. On interaction of polymer abrasive brush fiber with the machined surface, Proceedings of Moscow State Technical University MAMI, v. 2 (8), pp. 184-187 (2009)

5. Yu. D. Abrashkevich, L.S. Pelevin, G.M. Machishin. A mechanism of interaction of polymeric abrasive fiber with the machined surface, MINTT-2011: Materials of the Third International Scientific and Practical Conference. Kherson: Kherson State Maritime Academy, v. 1, pp. 104-108 (2011)

6. Yu. V. Dimov, D.B. Podashev. Edge forces in machining by abrasive brushes, The messenger of mechanical engineering (to be published)

7. Yu. V. Dimov, D.B. Podashev. Edge machining on sloping surfaces, The messenger of mechanical engineering (to be published)

8. Yu. V. Dimov, D.B. Podashev. Studies in the surface quality when edge rounding with abrasive brushes, Bulletin of Irkutsk National Research Technical University, v. 9, pp. 2834 (2016) 